\title{
Risk of Falls, Injurious Falls, and Other Injuries Resulting from Visual Impairment among Older Adults with Age-Related Macular Degeneration
}

\author{
Joanne M. Wood, ${ }^{1,2}$ Pbilippe Lacherez, ${ }^{1,2}$ Alex A. Black, ${ }^{1,2}$ Michael H. Cole, ${ }^{2,3,4}$ \\ Mei Ying Boon, ${ }^{1,2,5}$ and Grabam K. Kerr ${ }^{2,3}$
}

Purpose. Age-related macular degeneration (AMD) is the leading cause of irreversible visual impairment among older adults. This study explored the relationship between AMD, fall risk, and other injuries and identified visual risk factors for these adverse events.

Methods. Participants included 76 community-dwelling individuals with a range of severity of AMD (mean age, 77.0 \pm 6.9 years). Baseline assessment included binocular visual acuity, contrast sensitivity, and merged visual fields. Participants completed monthly falls and injury diaries for 1 year after the baseline assessment.

Results. Overall, $74 \%$ of participants reported having either a fall or a non-fall-related injury. Fifty-four percent of participants reported a fall and $30 \%$ reported more than one fall; of the 102 falls reported, 63\% resulted in an injury. Most occurred outdoors (52\%), between late morning and late afternoon (61\%) and when navigating on level ground (62\%). The most common non-fall-related injuries were lacerations (36\%) and collisions with an object (35\%). Reduced contrast sensitivity and visual acuity were associated with increased fall rate, after controlling for age, sex, cognitive function, cataract severity, and self-reported physical function. Reduced contrast sensitivity was the only significant predictor of non-fall-related injuries.

Conclusions. Among older adults with AMD, increased visual impairment was significantly associated with an increased incidence of falls and other injuries. Reduced contrast sensitivity was significantly associated with both increased rates of falls and other injuries, while reduced visual acuity was only associated with increased fall rate. These findings have important implications for the assessment of visually impaired older adults. (Invest Ophthalmol Vis Sci. 2011;52:5088-5092) DOI: 10.1167/iovs. 10-6644

O der adults have one of the highest injury-related mortality rates $^{1,2}$ and have a poorer prognosis and more complications after injury than their younger counterparts, ${ }^{3}$ which has

From the ${ }^{1}$ School of Optometry, ${ }^{2}$ Institute of Health \& Biomedical Innovation, and ${ }^{3}$ School of Human Movement Studies, Queensland University of Technology, Brisbane, Queensland, Australia; ${ }^{4}$ School of Exercise Science, Australian Catholic University, Brisbane, Queensland, Australia; and ${ }^{5}$ School of Optometry and Vision Science, University of New South Wales, Sydney, New South Wales, Australia.

Supported by the NHMRC Prevention of Injuries in Older People Partnership in Injury grant.

Submitted for publication September 27, 2010; revised February 9, 2011; accepted March 3, 2011.

Disclosure: J.M. Wood, None; P. Lacherez, None; A.A. Black, None; M.H. Cole, None; M.Y. Boon, None; G.K. Kerr, None

Corresponding author: Joanne M. Wood, School of Optometry and Institute of Health and Biomedical Innovation, QUT, Kelvin Grove, Brisbane, Q 4059, Australia; j.wood@qut.edu.au. significant ramifications for associated health care costs. ${ }^{4}$ It has been demonstrated that the risk of unintentional injury, especially resulting from falls, is higher for individuals with visual impairment compared with those with normal vision. ${ }^{5-7}$ However, little is known about the relationship between visual impairment and injuries unrelated to falls, such as lacerations and burns. ${ }^{8}$

Of particular relevance are the effects of visual impairment resulting from age-related macular degeneration (AMD) on falls and other injuries, given that AMD is the most common cause of irreversible visual impairment in older adults. Age-related macular degeneration affects a range of visual functions that have been associated with increased fall risk, including visual acuity, ${ }^{5,9-12}$ contrast sensitivity, ${ }^{13}$ and visual fields. ${ }^{6,10}$ There have also been numerous studies that have suggested that AMD is associated with impaired postural stability, ${ }^{14,15}$ mobility and gait, ${ }^{16-19}$ and a greater physiological fall risk profile. ${ }^{20}$ Some of these studies have also demonstrated that visual measures including contrast sensitivity and visual fields ${ }^{18,19,21}$ are better predictors of impaired balance and mobility performance in AMD than visual acuity. Despite these associations, only a limited number of studies have explored the relationship between AMD and falls, and none have identified the visual risk factors for falls and injuries specifically in this population.

Although earlier studies failed to find an association between AMD and increased fall risk, ${ }^{9,22,23}$ possibly because of insufficient numbers of AMD patients in the study populations, a more recent study reported that the risk of injurious falls was doubled in older women with neovascular AMD compared with age-matched controls. ${ }^{24}$ Importantly, the mean best eye visual acuity of the population at baseline was 20/80, representing a relatively advanced level of $\mathrm{AMD}$, and other visual function measures were not reported. It is thus not possible to identify which visual function measures best predicted falls in this population, information that is critical for eye care providers.

In this study we aimed to better understand the prospective injury risk from falls and non-fall-related causes over a 12month follow-up period in a sample of older adults with a range of levels of visual impairment due to AMD. In particular we were interested in identifying which visual factors best predicted prospective injury and falls in this population.

\section{Methods \\ Participants}

Eighty community dwelling older adults with retinal changes consistent with AMD who had no significant ocular or visual pathway disease leading to visual field loss, other than AMD, were included in the study and have been described in detail elsewhere. ${ }^{19}$ Participants were recruited from the School of Optometry Clinic at the Queensland 
University of Technology, via the electoral roll, or from Brisbane-based members of the Macular Degeneration Foundation (Sydney, Australia).

Participants were excluded from the study if they were unable to walk unaided or had a history of Parkinson's disease, diabetes, or peripheral neuropathy. Participants were also screened for cognitive impairment using the Mini Mental State Examination (MMSE). ${ }^{25}$ A small number of participants were unable to complete three of the $30 \mathrm{MMSE}$ items due to visual impairment (vision-based items ["CLOSE YOUR EYEs"], sentence writing, and design for copy) even with enlarged versions of these items (equivalent to 20/400); for these participants the relevant items were scored as correct. ${ }^{25,26}$ Participants with any comorbid eye disease (other than AMD or cataract) were not included in the study.

The research followed the tenets of the Declaration of Helsinki, and informed consent was obtained before participant assessment. The research was approved by the Queensland University of Technology Human Research Ethics Committee.

\section{Vision Assessment}

All participants attended a baseline assessment where they underwent an eye examination, including assessment of the presence and severity of lens opacities, using the slit lamp-based Lens Opacities Classification System (LOCSIII), ${ }^{27}$ and nonmydriatic, $45^{\circ}$ slide photography of the posterior pole using a fundus camera (Canon CR6-45NM; Canon, Tokyo, Japan) to confirm the presence of retinal changes consistent with AMD. The severity of AMD was also independently graded from the fundus slide photographs, according to the AREDS classification scheme. ${ }^{28}$ For the purpose of analysis, the highest LOCS score (either nuclear, posterior subcapsular, or cortical) in the eye with the better visual acuity was used as the level of cataract severity.

Binocular high-contrast visual acuity was measured with participants' habitual distance refractive correction using a Bailey-Lovie highcontrast letter chart at a working distance of $3.2 \mathrm{~m}$ and an average luminance of $195 \mathrm{~cd} / \mathrm{m}^{2}$. Participants were instructed to guess letters, even when they were unsure, until a full line of letters was incorrectly read. Visual acuity was scored letter by letter (each letter corresponded to $0.02 \log$ MAR units). Contrast sensitivity was measured binocularly using the paper version of the Melbourne Edge Test (MET), ${ }^{29}$ at a working distance of $40 \mathrm{~cm}$ and an average luminance of $65.5 \mathrm{~cd} / \mathrm{m}^{2}$, with an appropriate near correction. The MET is a four alternative forced-choice edge detection test that measures contrast sensitivity at around $3 \mathrm{c} / \mathrm{deg},{ }^{29}$ has excellent test-retest reliability, ${ }^{30}$ and has been used in a range of previous studies of vision and falls. ${ }^{31,32}$ Participants were asked to identify the orientation of the edge within each circular patch until two consecutive incorrect responses were made, and the lowest contrast edge correctly identified was recorded as the participant's contrast sensitivity in $\mathrm{dB}$.

Visual fields were assessed using the Humphrey Field Analyzer (Model HFA-II 750, Carl Zeiss Meditec, Dublin, CA). Monocular 24-2 SITA-Standard threshold tests were performed by an experienced optometrist. Where required, a large fixation target was used to ensure stable fixation during testing; in some cases where fixation errors were still high, fixation monitoring was disabled, and fixation was continuously monitored visually by the examiner. Field tests were considered reliable if false-positive and false-negative errors were $<33 \%$. ${ }^{33} \mathrm{~A}$ binocular mean deviation score was derived by merging the right and left fields to create an integrated binocular visual field, based on the more sensitive of the two eyes at each visual field location ${ }^{34}$; the binocular field was also considered separately as field loss at locations above (superior to) and below (inferior to) the midline. Participants' habitual correction and spectacle type used for walking (e.g., multifocal, single vision, progressive) were also recorded.

\section{Questionnaire}

The SF-36 physical function scale was used as a self-reported measure of physical function. This provides an index of general physical functioning and health and has been shown to be an effective and valid health care measure in older community-based populations. ${ }^{35}$

\section{Falls and Injury Diaries}

After the baseline assessment, participants were asked to complete a monthly diary that recorded any falls and other injuries experienced over that period and to return the diary in the prepaid envelopes provided. A fall was defined as unintentionally coming to the ground or some lower level not as a result of a major intrinsic event (e.g., stroke) or overwhelming hazard ${ }^{36}$; participants were asked to detail any injuries resulting from any falls. Participants were asked to record which spectacle correction they were wearing at the time of the fall. The non-fall-related injuries included burns or scalds, collision with an object, collision and laceration, eye injury, laceration, lifting and twisting injury, pedestrian accident, or sporting injury. If participants failed to complete their monthly diaries, they were sent reminders by mail and contacted by telephone.

\section{Statistical Analyses}

Statistical analyses were performed using commercially available software (SPSS v. 17.0 for Windows; SPSS, Chicago, IL). Because falls accounted for the majority of adverse events incurred in this sample, the events were separated into fall and non-fall-related events for analysis, and injurious falls were also considered separately for comparison. The number of falls, injurious falls, and non-fall-related injuries per participant during the 12-month period were examined using both Poisson and negative binomial regressions, with the Lagrange multiplier test used to test for overdispersion. The distribution of non-fall-related injuries revealed significant overdispersion, and therefore the negative binomial model was used, while falls and injurious falls did not reveal significant overdispersion, and therefore the Poisson model was retained as is recommended. ${ }^{37}$ For each outcome measure (falls, injurious falls, and other injuries), each visual function measure (visual acuity, contrast sensitivity, total mean deviation, superior mean deviation, inferior mean deviation) was examined in separate regression models. Separate models were conducted for each visual function measure because the visual measures were so highly correlated as to cause serious multicollinearity if they had been included in a simultaneous model (with correlations of around 0.7-0.8). All analyses controlled for the following potential confounding variables: age, MMSE, sex, physical function, and cataract severity; none of these variables were significantly related to any of the outcome measures in the models.

\section{Results}

Of the participants assessed at baseline, three participants did not complete any diaries, and a fourth completed only the first two diaries, and these were therefore not included in the analyses. The final sample consisted of 76 participants (mean age, $77.0 \pm 6.9$ years; range, $59-95$ years) including 34 males (45\%) and 42 females (55\%). Participants had a range of severity of AMD, in terms of both visual function and their AREDs grades, with more participants exhibiting binocular visual loss and AREDS scores in the mild to moderate rather than the severe categories (Table 1). For the better eye, 24 participants (32\%) were pseudophakic, $7(9 \%)$ had a LOCS score of 0,10 $(13 \%)$ had a grade of $1,17(23 \%)$ a grade of $2,10(13 \%)$ a grade of $3,7(9 \%)$ a grade of 4 , and $1(1 \%)$ a grade of 5 .

Overall, $74 \%(n=56)$ of the sample reported at least one fall or injury event during the 12 month follow-up period. Falls were the most common event, with $54 \%$ of the participants $(n=41)$ reporting at least one fall and $30 \%(n=23)$ reporting two or more falls. Seventeen participants reported three or more falls, with the highest incidence being eight during the one year follow-up period. Table 2 shows the characteristics of the reported falls and other injury events sustained by the sample over the follow-up period. Sixty-three percent of the falls $(n=64)$ resulted in an injury, but only $17 \%$ required medical treatment. Most falls occurred outdoors $(52 \%$ of all 
TABLE 1. Summary of the Visual Characteristics and AREDs Scores of Participants

\begin{tabular}{lc}
\hline \multicolumn{1}{c}{ Vision Characteristic } \\
\hline Binocular visual acuity, logMAR \\
$\quad$ Mean $\pm \mathrm{SD}$ \\
$\leq 0.3(20 / 40), n(\%)$ & $0.28 \pm 0.4$ \\
$>0.3(20 / 40)$ and $\leq 1(20 / 200), n(\%)$ & $18(67.1)$ \\
$>1(20 / 200), n(\%)$ & $7(9.2)$ \\
Binocular contrast sensitivity, dB & $16.61 \pm 4.52$ \\
Mean $\pm \mathrm{SD}$ & $41(53.9)$ \\
$\geq 18 \mathrm{~dB}, n(\%)$ & $23(30.3)$ \\
$<18 \mathrm{~dB}$ and $\geq 12 \mathrm{~dB}, n(\%)$ & $12(15.8)$ \\
$<12 \mathrm{~dB}, n(\%)$ & \\
Integrated visual fields, mean deviation, dB & $2.79 \pm 4.5$ \\
Mean $\pm \mathrm{SD}$ & $44(57.9)$ \\
$\geq-2 \mathrm{~dB}, n(\%)$ & $19(25)$ \\
$<-2 \mathrm{~dB}$ and $\geq-6 \mathrm{~dB}, n$ (\%) & $13(17.1)$ \\
$<-6 \mathrm{~dB}, n$ (\%) & \\
AREDS score (average of both eyes) & $2.32 \pm 1.2$ \\
Mean $\pm \mathrm{SD}$ & $26(34)$ \\
Early $(<2), n$ (\%) & $35(46)$ \\
Moderate $(\geq 2$ and $<4), n(\%)$ & $14(19)$ \\
Advanced (grade 4$), n(\%)$ & $1(1)$ \\
Missing, $n(\%)$ & \\
\hline
\end{tabular}

falls compared to $35 \%$ indoors and $13 \%$ unspecified), between late morning and late afternoon (61\%), and overall, falls occurred more often when navigating on level ground (62\% of all falls) than when rising or reclining. The main reason reported for a fall was tripping ( $40 \%)$, followed by loss of balance (25\%), with misplaced stepping being the least likely reason reported for falling (9\%). We also examined whether falls were associated with the use of multifocal spectacles. The use of multifocal spectacles correction did not differ significantly between fallers and nonfallers $\left(57 \%\right.$ and $59 \%$, respectively, $\chi^{2}[1]=$ $0.015, P=0.9)$, or between multiple fallers and nonmultiple fallers $\left(60 \%\right.$ and $\left.52 \%, \chi^{2}[1]=0.433, P=0.506\right)$. Interestingly, $21 \%$ of those who fell were not wearing their habitual spectacle correction at the time of the fall.

There were 138 non-fall-related injuries, of which lacerations (36\%) and collisions with an object (35\%) were most common, followed by lifting or twisting injuries, and then burn or scald injuries (Table 2).

Table 3 shows the results of a series of regression analyses predicting falls and injury rates based on each of the visual function measures, controlling for age, MMSE, sex, physical function, and cataract severity. Reduced contrast sensitivity and visual acuity were significantly associated with an increased rate of falls. When only injurious falls were considered, reduced contrast sensitivity and visual acuity were still the only significant visual predictors. However, only reduced contrast sensitivity was significantly associated with an increased rate of other injury events.

\section{Discussion}

This study demonstrated among older adults with AMD who were free of ocular disease other than cataract that the presence of increased visual impairment is associated with a higher incidence of prospective falls and injuries recorded over a 12 month follow-up period. Of the visual function measures assessed, reduced contrast sensitivity was significantly associated with an increased rate of both falls and other injuries, while reduced visual acuity was significantly associated only with an increase in falls. Visual field loss within the central $24^{\circ}$ was not significantly associated with either falls or other injuries in this sample.
The finding that contrast sensitivity was the strongest visual predictor of falls among older adults with AMD is in accord with previous studies that have demonstrated associations between reduced contrast sensitivity and increased postural sway on foam ${ }^{14,19}$ and gait adaptations in older adults with AMD. ${ }^{18,19,21}$ The findings also support those of general population studies where fall risk has been shown to be increased in the presence of reduced contrast sensitivity. ${ }^{13,38}$ Avoidance of trip hazards and negotiating stairs are likely to be reliant on adequate visual information over a range of spatial frequencies, and hence measures of contrast sensitivity better predicted these adverse events in this population. The finding that reduced contrast sensitivity also predicts the propensity for other injuries is also highly relevant given that older adults in general, regardless of their visual status, have high injury-related mor-

TABle 2. Characteristics of Prospective Falls and Injuries Sustained over the 12-Month Follow-up Period

\begin{tabular}{lrr}
\hline & $\boldsymbol{n}$ & $\%$ \\
\hline All falls & 102 & 100 \\
Injurious falls & 64 & 63 \\
Injury sustained & & \\
$\quad$ Soft-tissue injury & 55 & 86 \\
$\quad$ Fracture & 4 & 6 \\
Head trauma & 5 & 8
\end{tabular}

Fall location

Inside

Inside own home, on the one level

Inside own home, walking up or down stairs

Inside but not own home, on the one level

Inside own home, getting out of a chair

Inside own home, getting out of bed

Inside own home, accessing the toilet

Inside but not own home, accessing the shower/bath

Inside own home, accessing the shower/bath

Outside

On the one level

Garden/park/grassed area

On a footpath

Of own home, walking up or down stairs

On the one level

On a bus/train

Car park/driveway

On a step/escalator

On a curb

Getting into or out of a vehicle Other

Cause of fall

Trip

Misplaced step

Slip

Loss of balance

Not reported

Fall time of day

Night time (6 PM-5:59 AM)

Early morning (6 AM-10:59 AM)

Midday (11 AM-2:59 PM)

Afternoon (3 PM-5:59 PM)

Not indicated

Other injuries

Laceration

Collision with object

Lifting or twisting injury

Burn or scald

Other

Sporting injury

Eye injury

Pedestrian accident

Collision and laceration

\section{0}

63

86

6
8

16

6

16

6

4

3

2

2

2

$<1$

16

10

9

6

3

2

2

2

2

$<1$

13

40

9

16

25

10

11

19

36

25

9

36

35

16

9

2

$<1$

$<1$

$<1$

$<1$ 
TABLE 3. Regression Predicting Prospective Falls and Injurious Falls (Poisson) and Other Injury (Negative Binomial) Based on Measures of Visual Function

\begin{tabular}{|c|c|c|c|c|c|c|c|c|c|}
\hline & \multicolumn{3}{|c|}{ Falls (Poisson) } & \multicolumn{3}{|c|}{ Injurious Falls (Poisson) } & \multicolumn{3}{|c|}{$\begin{array}{l}\text { Other Injury Events } \\
\text { (Negative Binomial) }\end{array}$} \\
\hline & $\mathbf{B}$ & $\begin{array}{l}\text { Wald } \chi^{2} \\
(1 \mathrm{df})\end{array}$ & $\boldsymbol{P}$ & $\mathbf{B}$ & $\begin{array}{l}\text { Wald } \chi^{2} \\
(1 \mathrm{df})\end{array}$ & $\boldsymbol{P}$ & $\mathbf{B}$ & $\begin{array}{l}\text { Wald } \chi^{2} \\
(1 \mathrm{df})\end{array}$ & $\boldsymbol{P}$ \\
\hline \multicolumn{10}{|c|}{ Binocular contrast sensitivity, Melbourne } \\
\hline Edge Test, dB & -0.092 & 13.332 & $<0.001$ & -0.081 & 6.031 & 0.014 & -0.087 & 4.361 & 0.037 \\
\hline Binocular visual acuity, logMAR & 0.631 & 6.947 & 0.008 & 0.629 & 4.173 & 0.041 & 0.377 & 0.831 & 0.362 \\
\hline \multicolumn{10}{|l|}{ Binocular visual fields, dB } \\
\hline Mean defect (total) & 0.009 & 0.123 & 0.726 & 0.048 & 1.675 & 0.196 & -0.012 & 0.092 & 0.762 \\
\hline Mean defect (superior) & 0.009 & 0.130 & 0.719 & 0.039 & 1.332 & 0.249 & -0.012 & 0.111 & 0.739 \\
\hline Mean defect (inferior) & 0.008 & 0.096 & 0.757 & 0.052 & 1.772 & 0.183 & -0.009 & 0.052 & 0.820 \\
\hline
\end{tabular}

Note: All analyses were performed controlling for age, MMSE, sex, physical function, and cataract severity. Each visual function measure was examined in a separate regression to avoid multicollinearity.

tality rates ${ }^{1}$ and have a worse prognosis and more complications after injury than their younger counterparts. ${ }^{3}$

Visual acuity was also associated with an increased rate of falls but not with increased risk for other injuries. The role of reduced visual acuity in increased fall risk is not unexpected given previous studies that have shown collectively that those with reduced visual acuity are 1.7 times more likely to have a fall and 1.9 times more likely to have multiple falls compared with those with normal vision. ${ }^{8}$ Although more recent general population studies have identified that visual field loss is the strongest visual predictor of falls, ${ }^{6,7}$ this was not found to be the case for this sample. This may be because in our sample the participants were free of ocular diseases other than AMD and early cataract and hence had localized central rather than peripheral field loss; in addition, measurement of visual fields in this population tends to be less accurate due to fixation difficulties. ${ }^{39}$ It is also important to note that the visual field measures in this study were confined to the central $24^{\circ}$ of the visual field rather than in more peripheral locations.

In this study, a high proportion of participants reported a fall during the 12-month follow-up period; $54 \%$ reported at least one fall, and $30 \%$ reported two or more falls. These rates are higher than those previously reported among general population studies of older adults, which have reported annual fall rates of around $30 \%{ }^{7}$ and annual multiple fall rates around $16 \%{ }^{6}$ Furthermore, our participants reported a mean of 0.84 injurious falls per person-year, which is higher than the rate of 0.37 injurious falls per person-year reported by a previous study of older women with neovascular AMD. ${ }^{24}$ It is difficult, however, to directly compare rates between studies given the considerable variations in study designs, fall and injury definitions, and sample populations.

Falls were more common between late morning to late afternoon and on level surfaces, however this may simply be because older adults are more likely to be out and about at this time, and most walking occurs on level surfaces. Falls also tended to occur more often when walking outdoors rather than indoors and were the result of tripping or loss of balance rather than a misplaced step.

When we examined the pattern of non-fall-related injuries, the largest proportion were lacerations, followed by collisions with an object, which is consistent with previous studies that have examined home-based injuries. ${ }^{2,40}$ Another study did not report collisions as a separate category, but in accord with our findings reported that lacerations were the most common non-fall-related injury. ${ }^{41}$ It is difficult to compare our results with those of studies that report on more serious injuries resulting in hospital admissions or death, ${ }^{42,43}$ given that they involved a higher degree of injury severity than that examined in the present study. Comparison of the rates of these falls and other injuries among larger populations of vision impaired and visually normal individuals would be informative.

Collectively, these findings have clinical implications for the management and advice that should be provided to patients with AMD and highlight the potential injury risks that these patients are exposed to in their daily activities, even when most of the sample had mild to moderate rather than severe levels of AMD. Importantly, contrast sensitivity was the strongest predictor of both the rates of falls and other injuries in this population. A larger-scale prospective study is required to establish the levels of AMD severity and visual impairment at which the injury risk begins to increase. An improved understanding of this would help eye care providers to identify at which stage patients should be referred to appropriate rehabilitation specialists for advice regarding modifiable risk factors (shoe wear, floor coverings, physical activity) and interventions, such as hip protectors, which reduce the risk of injury in the event of a fall. ${ }^{44}$

This study has a number of strengths including the use of prospective falls as one of our primary outcome measures, which are now considered the gold standard for recording falls rather than retrospective falls measures. ${ }^{45}$ We adopted a similar prospective approach to recording other injury events. Furthermore, the study used a standardized battery of visual function measures that incorporated commercially available instrumentation, allowing replication of the study findings and application in a range of settings. The most important limitation of the study was the relatively small sample size, and future studies should include larger cohorts of patients with varying levels of visual impairment from AMD. Nevertheless, these preliminary findings suggest that reductions in contrast sensitivity are the most important predictors of increased risk of falls and other injuries in this population.

In summary, this study highlights the importance of contrast sensitivity screening among older adults with AMD, using easy-to-administer and reliable contrast sensitivity measures such as the MET. These measures can be performed by appropriately trained personnel in eye and health care settings and will allow clinicians to identify those older adults with AMD who are at risk of future falls and injuries.

\section{Acknowledgments}

The authors thank The Macular Degeneration Foundation (Sydney, Australia) for their assistance in recruiting participants to this study, all the participants who gave so generously of their time, and Roger Martin, Melissa Newton, Matthew Roodveldt, Jocelyn Stewart, Oren 
Tirosh, and Paul Turner for assistance in various stages of the laboratory-based data collection.

\section{References}

1. Lilley JM, Arie T, Chilvers CE. Accidents involving older people: a review of the literature. Age Ageing. 1995;24:346-365.

2. Xiang $\mathrm{H}$, Leff $M$, Stallones L. Non-fatal injuries among adults with activity limitations and participation restrictions. Inj Prev. 2005; 11:157-162.

3. van der Sluis CK, Klasen HJ, Eisma WH, ten Duis HJ. Major trauma in young and old: what is the difference? J Trauma. 1996; $40: 78-82$.

4. Covington DL, Maxwell JG, Clancy TV. Hospital resources used to treat the injured elderly at North Carolina trauma centers. $J$ Am Geriatr Soc. 1993;41:847-852.

5. Klein BE, Klein R, Lee KE, Cruickshanks KJ. Performance-based and self-assessed measures of visual function as related to history of falls, hip fractures, and measured gait time. The Beaver Dam Eye Study. Opbthalmology. 1998;105:160-164.

6. Coleman AL, Cummings SR, Yu F, et al. Binocular visual-field loss increases the risk of future falls in older white women. $\mathrm{J} \mathrm{Am}$ Geriatr Soc. 2007;55:357-364.

7. Freeman EE, Munoz B, Rubin G, West SK. Visual field loss increases the risk of falls in older adults: the Salisbury eye evaluation. Invest Ophthalmol Vis Sci. 2007;48:4445-4450.

8. Legood R, Scuffham P, Cryer C. Are we blind to injuries in the visually impaired? A review of the literature. Inj Prev. 2002;8:155160.

9. Ivers RQ, Cumming RG, Mitchell P, Attebo K. Visual impairment and falls in older adults: the Blue Mountains Eye Study. $\mathrm{J} \mathrm{Am}$ Geriatr Soc. 1998;46:58-64.

10. Klein BE, Moss SE, Klein R, Lee KE, Cruickshanks KJ. Associations of visual function with physical outcomes and limitations 5 years later in an older population: the Beaver Dam eye study. Ophthalmology. 2003;110:644-650

11. Lord SR. Visual risk factors for falls in older people. Age Ageing. 2006;35(Suppl 2):ii42-ii45.

12. Coleman AL, Stone K, Ewing SK, et al. Higher risk of multiple falls among elderly women who lose visual acuity. Ophthalmology 2004; 111:857-862.

13. de Boer MR, Pluijm SM, Lips P, et al. Different aspects of visual impairment as risk factors for falls and fractures in older men and women. J Bone Miner Res. 2004;19:1539-1547.

14. Elliott DB, Patla AE, Flanagan JG, et al. The Waterloo Vision and Mobility Study: postural control strategies in subjects with ARM. Ophthal Phys Opt. 1995;15:553-559.

15. Turano KA, Dagnelie G, Herdman SJ. Visual stabilization of posture in persons with central visual field loss. Invest Ophthalmol Vis Sci. 1996;37:1483-1491.

16. Spaulding SJ, Patla AE, Elliott DB, Flanagan J, Rietdyk S, Brown KS Waterloo Vision and Mobility Study: gait adaptations to altered surfaces in individuals with age-related maculopathy. Optom Vis Sci. 1994;71:770-777.

17. Spaulding SJ, Patla AE, Flanagan J, Elliott DB, Rietdyk S, Brown KS Waterloo Vision and Mobility Study: normal gait characteristics during dark and light adaptation in individuals with age-related maculopathy. Gait \& Post. 1995;3:227-235.

18. Kuyk T, Elliott JL. Visual factors and mobility in persons with age-related macular degeneration. J Rehab Res Dev. 1999;36:303312 .

19. Wood JM, Lacherez PF, Black AA, Cole MH, Boon MY, Kerr GK Postural stability and gait among older adults with age-related maculopathy. Invest Ophthalmol Vis Sci. 2009;50:482-487.

20. Szabo SM, Janssen PA, Khan K, Potter MJ, Lord SR. Older women with age-related macular degeneration have a greater risk of falls: a physiological profile assessment study. J Am Geriatr Soc. 2008; 56:800-807.

21. Hassan SE, Lovie-Kitchin JE, Woods RL. Vision and mobility per formance of subjects with age-related macular degeneration. $O p$ tom Vis Sci. 2002;79:697-707.
22. Felson DT, Anderson JJ, Hannan MT, Milton RC, Wilson PW, Kiel DP. Impaired vision and hip fracture. The Framingham Study. J Am Geriatr Soc. 1989;37:495-500.

23. Ivers RQ, Cumming RG, Mitchell P, Simpson JM, Peduto AJ. Visual risk factors for hip fracture in older people. J Am Geriatr Soc. 2003;51:356-363.

24. Szabo SM, Janssen PA, Khan K, Lord SR, Potter MJ. Neovascular AMD: an overlooked risk factor for injurious falls. Osteoporos Int. 2010;21:855-862.

25. Folstein MF, Folstein SE, McHugh PR. Mini-mental state: a practical method for grading the cognitive state of patients for the clinician. J Psych Res. 1975;12:189-198.

26. Heun R, Papassotiropoulos A, Jennssen F. The validity of psychometric instruments for detection of dementia in the elderly general population. Int J Ger Psych. 1998;13:368-380.

27. Chylack LT, Jr., Wolfe JK, Singer DM, et al. The Lens Opacities Classification System III. The Longitudinal Study of Cataract Study Group. Arch Opbthal. 1993;111:831-836.

28. The Age-Related Eye Disease Research Group. The Age-Related Eye Disease Study (AREDS) system for classifying age-related macular degeneration from stereoscopic color fundus photographs: AREDS Report No. 6. Am J Opbthalmol 2001;132:668-681.

29. Verbaken JH, Johnston AW. Population norms for edge contrast sensitivity. Am J Optom Physiol Opt. 1986;63:724-732.

30. Haymes SA, Chen J. Reliability and validity of the Melbourne Edge Test and High/Low Contrast Visual Acuity chart. Optom Vis Sci. 2004;81:308-316.

31. Lord SR, Menz HB. Visual contributions to postural stability in older adults. Gerontology. 2000;46:306-310.

32. Lord SR, Dayhew J. Visual risk factors for falls in older people. J Am Geriatr Soc. 2001;49:508-515.

33. Johnson CA, Keltner JL, Cello KE, et al. Baseline visual field characteristics in the ocular hypertension treatment study. Ophthalmology. 2002;109:432-437.

34. Nelson-Quigg JM, Cello K, Johnson CA. Predicting binocular visual field sensitivity from monocular visual field results. Invest $O p b$ thalmol Vis Sci. 2000;41:2212-2221.

35. Chia EM, Chia EM, Rochtchina E, Wang JJ, Mitchell P. Utility and validity of the self-administered SF-36: findings from an older population. Ann Acad Med Singapore 2006;35:461-467.

36. Lord SR, Menz HB, Tiedemann A. A physiological profile approach to falls risk assessment and prevention. Phys Ther. 2003;83:237252.

37. Orme JG, Combes-Orme T. Multiple Regression with Discrete Dependent Variables. Oxford: Oxford University Press; 2009.

38. Cummings SR, Nevitt MC, Browner WS, et al. Risk factors for hip fracture in white women. Study of Osteoporotic Fractures Research Group. N Engl J Med. 1995;332:767-773.

39. Almog Y, Shiftan A. Plotting visual fields using contralateral fixation: a novel honeycomb device. Arch Opbthalmol. 2004;122: 1052-1056.

40. Graham HJ, Firth J. Home accidents in older people: role of primary health care team. BMJ. 1992;305:30-32.

41. Carter SE, Campbell EM, Sanson-Fisher RW, Gillespie WJ. Accidents in older people living at home: a community-based study assessing prevalence, type, location and injuries. Aust NZJ Public Health. 2000;24:633-636.

42. Wolf ME, Rivara FP. Nonfall injuries in older adults. Annu Rev Public Health. 1992;13:509-528.

43. $\mathrm{Hu} \mathrm{G}$, Baker SP. Recent increases in fatal and non-fatal injury among people aged 65 years and over in the USA. Inj Prev. 2010;16:26-30.

44. Parker MJ, Gillespie WJ, Gillespie LD. Hip protectors for preventing hip fractures in older people. Cochrane Database Syst Rev. 2005;CD001255.

45. Lamb SE, Jorstad-Stein EC, Hauer K, Becker C. Development of a common outcome data set for fall injury prevention trials: the Prevention of Falls Network Europe consensus. J Am Geriatr Soc. 2005;53:1618-1622. 